\title{
Alterations in aortic elasticity in noncompaction cardiomyopathy
}

\author{
Attila Nemes · Kadir Caliskan • \\ Marcel L. Geleijnse · Osama I. I. Soliman • \\ Ashraf M. Anwar · Folkert J. ten Cate
}

Received: 11 October 2006/ Accepted: 6 February 2007/Published online: 3 March 2007

(C) Springer Science+Business Media B.V. 2007

\begin{abstract}
Background Noncompaction cardiomyopathy (NCCM) is a recently recognized disorder frequently associated with systolic and diastolic heart failures. This study was designed to examine aortic stiffness in NCCM patients and to compare these results to age- and gender-matched controls.

Methods A total of 20 patients with typical echocardiographic features of NCCM (age $38 \pm 16$ years, eight males) were investigated. Their results were compared to 20 age- and gender-matched controls. All subjects underwent a complete two-dimensional transthoracic echocardiographic examination. Systolic (SD) and diastolic (DD) ascending aortic diameters were recorded in M-mode at a level of $3 \mathrm{~cm}$ above the aortic valve from a parasternal long-axis view. Aortic stiffness index $(\beta)$ was calculated as a
\end{abstract}

Attila Nemes is a visiting fellow from the University of Szeged (Szeged, Hungary) and is supported by the Research Fellowship of the European Society of Cardiology.Osama I.I. Soliman and Ashraf M. Anwar are visiting fellows from the Al Azhar University (Cairo, Egypt) and are supported by the Egyptian Government.

A. Nemes · K. Caliskan · M. L. Geleijnse .

O. I. I. Soliman · A. M. Anwar · F. J. ten Cate ( $₫)$ Department of Cardiology, Thoraxcenter, Erasmus Medical Center Rotterdam, Dr. Molewaterplein 40, 3015 GD, Room Ba304, Rotterdam, The Netherlands e-mail: f.j.tencate@erasmusmc.nl characteristic of aortic elasticity, as $\ln (\mathrm{SBP} / \mathrm{DBP}) /$ [(SD - DD)/DD], where SBP and DBP are the systolic and diastolic blood pressures, respectively, and $\ln$ is the natural logarithm.

Results The number of noncompacted segments in the NCCM patients was $4.6 \pm 2.0$. NCCM patients had significantly increased left ventricular dimensions and reduced left ventricular ejection fraction. Compared to controls, aortic stiffness index $(\beta)$ was significantly increased in $\mathrm{NCCM}$ patients $(8.3 \pm 5.2 \quad$ vs. $3.5 \pm 1.1$, $p<0.001)$.

Conclusion Increased aortic stiffness can be observed in patients with NCCM with moderate to severe heart failure. These alterations may be due to neurohormonal changes in heart failure.

Keywords Echocardiography $\cdot$ Noncompaction · Aortic stiffness

\section{Introduction}

Noncompaction cardiomyopathy (NCCM) is a recently recognized rare disorder [1, 2]. It is characterized by prominent myocardial trabecularizations, and deep intertrabecular recesses leading to the spongy appearance of the myocardium [3]. The disease is frequently associated with systolic and diastolic heart failure (HF), ventricular arrhythmias, and systemic embolization [4]. 
Alterations in arterial function have been demonstrated in patients with $\operatorname{HF}[5,6]$. With two-dimensional transthoracic echocardiography (TTE), ascending aortic diameter changes during a heart cycle can be measured. When blood pressure data are also available, aortic elasticity can be characterized [7, 8]. This study was designed to examine aortic stiffness in NCCM patients and to compare these results to age- and gender-matched controls.

\section{Methods}

Study population

A total of 20 patients with typical echocardiographic features of NCCM were investigated [3, 9]. Their clinical and demographic data are presented in Table 1. Clinical assessment included medical and family history, physical examination, electrocardiography, two-dimensional echocardiography, and, in most cases, contrast echocardiography. Their results were compared to 20 age- and gender-matched controls without apparent cardiovascular disease. Informed consent was obtained from each patient, and the study was approved by the institutional review board and complied with the Declaration of Helsinki.

Table 1. Clinical and demographic data of NCCM patients

\begin{tabular}{ll}
\hline & NCCM patients \\
\hline Age (years) & $38 \pm 16$ \\
Male (\%) & $8(40)$ \\
Diabetes (\%) & $1(5)$ \\
Index event & \\
Arrhythmia (\%) & $6(30)$ \\
Heart failure (\%) & $8(40)$ \\
Screening (\%) & $6(30)$ \\
Electrocardiogram & \\
Atrial fibrillation (\%) & $2(10)$ \\
LV hypertrophy (\%) & $3(15)$ \\
Left bundle branch block (\%) & $6(30)$ \\
\hline
\end{tabular}

NCCM noncompaction cardiomyopathy
Diagnostic criteria for NCCM

Previously proposed echocardiographic diagnostic criteria for NCCM by Jenni et al. [3] were used: (1) absence of coexisting cardiac anomalies, (2) segmental, excessive thickening of the left ventricular (LV) wall with a two-layered structure: a thin, compacted epicardial layer and a much thicker, noncompacted layer with the characteristic appearance of numerous, prominent trabeculations (meshwork) and deep intertrabecular recesses, (3) color Doppler evidence of deeply perfused intertrabecular recesses, and (4) predominant localization of thickening in the LV apical, midlateral, and midinferior walls. Hypertensive heart disease was excluded by clinical and echocardiographic examinations (septal thickness $<13 \mathrm{~mm}$ ).

Blood pressure measurement

Systolic and diastolic blood pressures (SBP and DBP, respectively) were measured in the supine position with an automatic mercury cuff sphygmomanometer from the left arm after 10 min of rest. The first and the fifth Koratkoff sounds were taken for the SBP and DBP. Blood pressure values were averaged from three consecutive measurements. None of the patients or controls used coffee or tea within $1 \mathrm{~h}$ before blood pressure measurements.

Transthoracic echocardiography

All patients underwent a complete two-dimensional TTE and Doppler study using a Philips Sonos 7500 echocardiography equipment (Philips, Best, The Netherlands) in the left lateral decubitus position from multiple windows. The LV wall segments were analyzed according to the 9-segment model as described by Jenni et al. [3]. Systolic and diastolic ascending aortic diameters (SD and DD, respectively) were recorded in M-mode at a level of $3 \mathrm{~cm}$ above the aortic valve from a parasternal long-axis view (Fig. 1). Gain, depth, and sector angles were individualized for the best measurement. The SD and DD were measured at the time of maximum anterior motion of the aorta and at the peak of the QRS complex, respectively. 


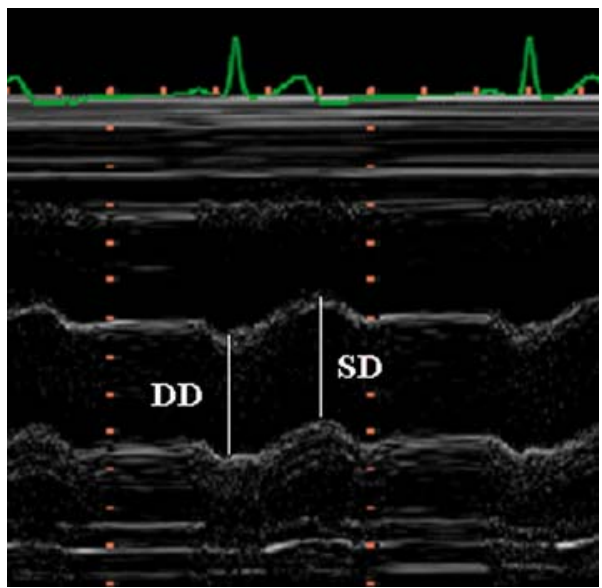

Fig. 1 Measurements of systolic $(S D)$ and diastolic $(D D)$ diameters of the ascending aorta are shown on the Mmode tracing obtained at a level $3 \mathrm{~cm}$ above the aortic valve

Evaluation of aortic stiffness

Aortic stiffness index $(\beta)$ was defined as $\ln (\mathrm{SBP} /$ $\mathrm{DBP}) /[(\mathrm{SD}-\mathrm{DD}) / \mathrm{DD}]$, where $\ln$ is the natural logarithm.

\section{Statistical analysis}

Data are reported as means \pm standard deviation. For variables, Student's $t$ test and analysis of variance (ANOVA) test were used where needed. A value of $p<0.05$ was considered statistically significant. SPSS 12.0 software was used for statistical calculations.
Reproducibility of echocardiographic measurements

The reproducibility of the aortic diameter measurements was tested in all NCCM patients at both systole and diastole by two independent, blinded observers. Agreement between the two observers was verified using the Bland-Altman method [10].

\section{Results}

\section{Patient population}

The presenting symptoms were HF in eight $(40 \%)$ and arrhythmias in six (30\%) patients. The remaining six $(30 \%)$ asymptomatic patients were NCCM relatives and were diagnosed after family screening (Table 1). Three HF patients were in NYHA-class III HF and the other five were in NYHA-class II. Cardiac medication used in NCCM patients were: angiotensin-converting enzyme inhibitors in $13(65 \%)$, beta-blockers in $11(55 \%)$, oral anticoagulant therapy in $10(50 \%)$, diuretics in $8(40 \%)$, digitalis in $2(10 \%)$, and nitrate in $1(5 \%)$. None of the controls received any cardiac medication.

\section{Transthoracic echocardiography}

The number of noncompacted segments in the NCCM patients was $4.6 \pm 2.0$ in the NCCM group and (as seen in Table 2) these patients had
Table 2. Transthoracic echocardiographic and blood pressure data in NCCM patients and normal subjects

Continuous variables are given as mean \pm standard deviation

$L V$ left ventricular, NCCM noncompaction cardiomyopathy * $p<0.001$

\begin{tabular}{lcc}
\hline & $\begin{array}{c}\text { Group } 1 \text { (normal } \\
\text { subjects) }\end{array}$ & $\begin{array}{c}\text { Group 2 (NCCM } \\
\text { patients) }\end{array}$ \\
\hline LV end-diastolic diameter $(\mathrm{mm})$ & $47.4 \pm 3.6$ & $61.0 \pm 10.9^{*}$ \\
LV end-systolic diameter $(\mathrm{mm})$ & $29.9 \pm 2.9$ & $48.2 \pm 12.3^{*}$ \\
LV ejection fraction $(\%)$ & $67.5 \pm 5.9$ & $36.0 \pm 17.6^{*}$ \\
Systolic aortic diameter $(\mathrm{mm})$ & $26.7 \pm 4.1$ & $26.6 \pm 4.4$ \\
Diastolic aortic diameter $(\mathrm{mm})$ & $23.3 \pm 3.8$ & $24.6 \pm 4.3$ \\
Pulsatile change in aortic diameter & $3.4 \pm 1.1$ & $2.0 \pm 1.2^{*}$ \\
$\quad($ mm) & $125.2 \pm 12.9$ & $120.4 \pm 17.4$ \\
Systolic blood pressure $(\mathrm{mmHg})$ & $77.8 \pm 8.5$ & $74.1 \pm 9.6$ \\
Diastolic blood pressure $(\mathrm{mmHg})$ & $47.4 \pm 8.7$ & $46.3 \pm 11.7$ \\
Aortic pulse pressure $(\mathrm{mmHg})$ & $3.5 \pm 1.1$ & $8.3 \pm 5.2^{*}$ \\
Aortic stiffness index $(\beta)$ & & \\
\hline
\end{tabular}


significantly increased LV dimensions and reduced LV ejection fraction. Aortic stiffness index $(\beta)$ was significantly increased in NCCM patients compared to controls. For controls, NCCM patients with moderate HF, and NCCM patients with severe $\mathrm{HF}, \beta$ values were $3.5 \pm 1.1$, $7.9 \pm 5.5$, and $10.4 \pm 1.8$, respectively $(p<0.001)$. Aortic stiffness indices of all individual patients and controls are presented in Fig. 2.

\section{Reproducibility}

The mean \pm standard deviation difference in values obtained by two observers for the measurements of aortic diameter at systole was $-0.7 \pm 2.2 \mathrm{~mm}$, with a correlation coefficient between these independent measurements of $0.88(p<0.01)$ (Figs. 3a and 4a). At diastole, the difference between these observations was $0.05 \pm 1.95 \mathrm{~mm}$, with a correlation coefficient between observations of $0.9(p<0.01)$. The difference in values that were detected by observers was within twofold of the standard deviation of the mean (Figs. $3 b$ and $4 b$ ).

\section{Discussion}

NCCM is a recently recognized disorder characterized by a loosened, spongy myocardium associated with a high incidence of progressive systolic and diastolic HF. In recent studies,

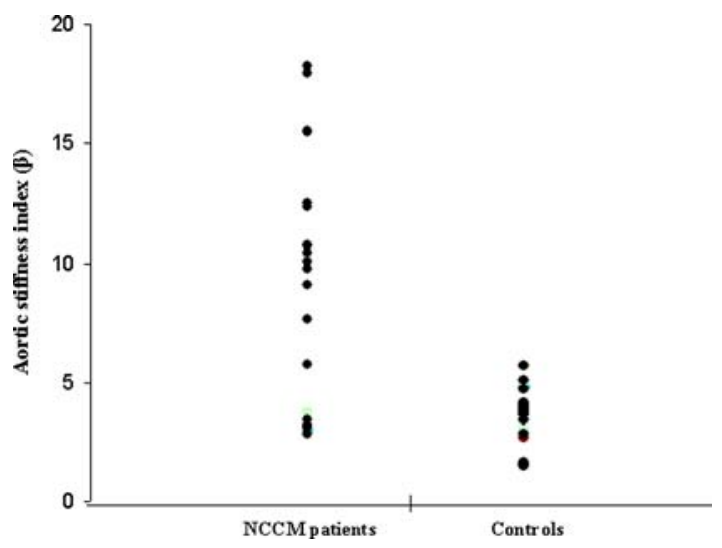

Fig. 2 Individual $\beta$ indices of NCCM patients and control subjects. NCCM noncompaction cardiomyopathy
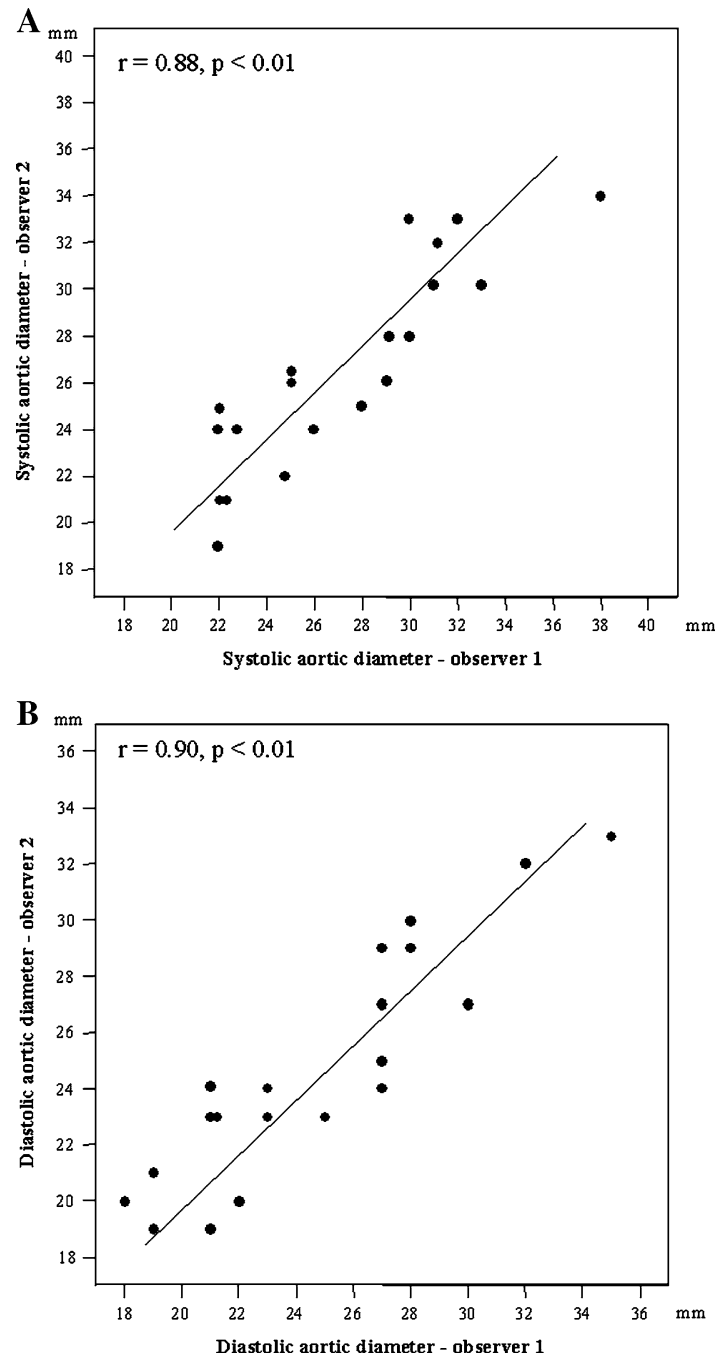

Fig. 3 a Interobserver correlation $(r=0.88, p<0.01)$ between aortic systolic diameters in NCCM patients. b Interobserver correlation $(r=0.9, p<0.01)$ between aortic diastolic diameters in NCCM patients. NCCM noncompaction cardiomyopathy

alterations have been demonstrated in arterial function in patients with chronic HF [11-14]. To the best of authors's knowledge, this is the first time that the aortic distensibility in a series of NCCM patients was examined. In this study, increased aortic stiffness index $(\beta)$ was found in NCCM patients compared to age- and gendermatched controls.

In prior studies, it has been shown that pulsatile changes in ascending aortic vessel diameter can be indirectly measured during routine TTE. Stefanadis et al. [7] found that noninvasive 
Fig. 4 a Reproducibility of systolic aortic measurements in NCCM patients. b

Reproducibility of diastolic aortic measurements in NCCM patients. NCCM noncompaction cardiomyopathy
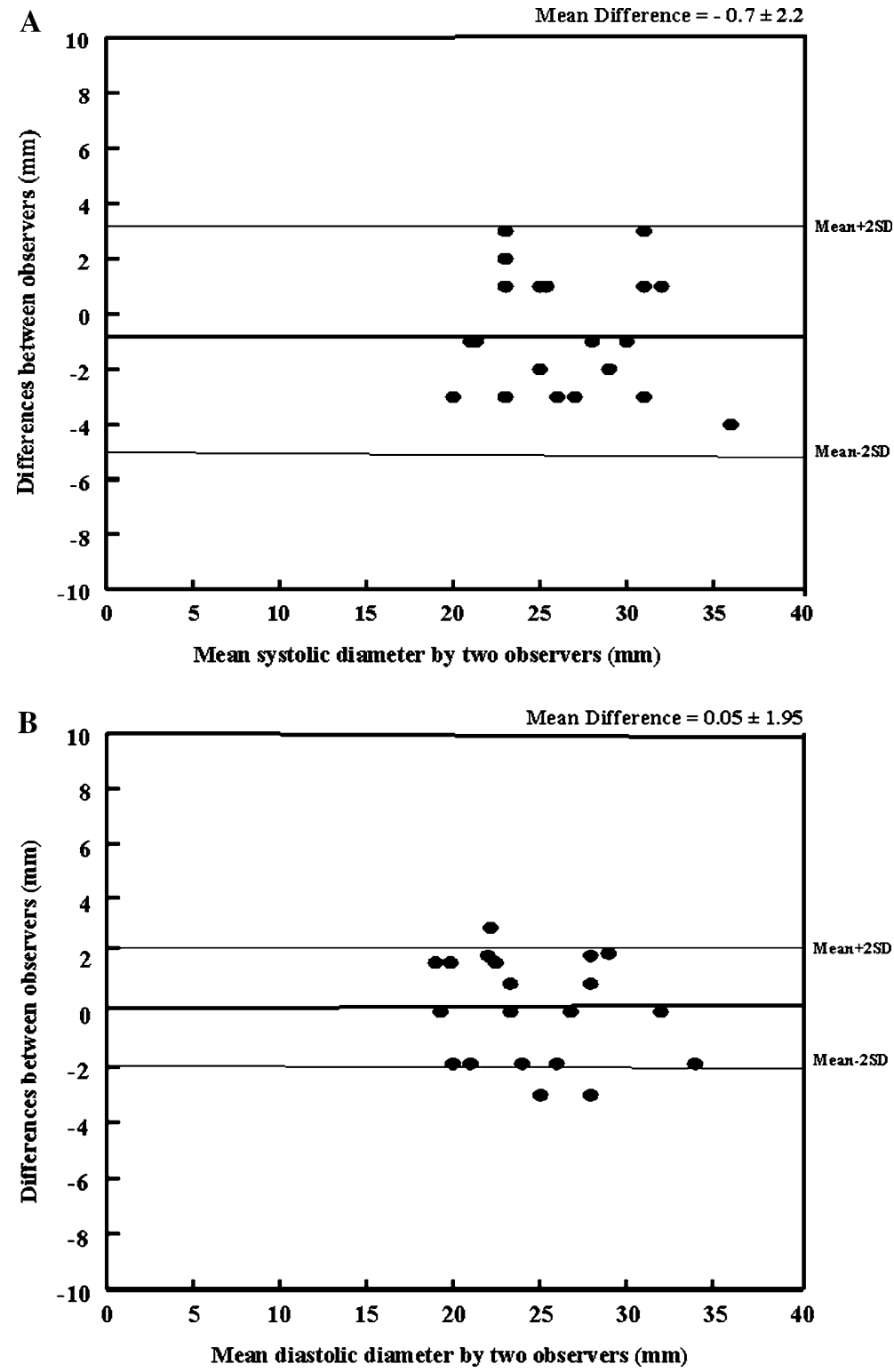

measurements of aortic distensibility were as accurate as invasive methods. Aortic stiffness index $(\beta)$ relies on aortic and blood pressure data and is one of the most frequently used parameters to characterize arterial stiffness [7, 8].

Increased aortic stiffness is an independent risk factor, predictor of cardiovascular mortality, and a contributor to $\mathrm{LV}$ afterload [15, 16]. Increased aortic stiffness occurs early during the development of pacing-induced congestive HF in animals
$[17,18]$ and has also been described in clinical patients with HF [5, 6], with comparable changes in arterial distensibility in ischemic and idiopathic dilated cardiomyopathy [19]. Rerkpattanapipat et al. [11] demonstrated that the distensibility of the proximal aorta is markedly reduced in older patients with HF due to LV systolic dysfunction beyond the changes from the aging process. They also described a correlation between exercise intolerance and reduced aortic distensibility. 
Giannattasio et al. [12] described that arterial compliance is impaired in congestive $\mathrm{HF}$, and although more marked in severe congestive HF, the impairment is manifested in mild congestive HF as well [12]. Nakamura et al. [13] described vascular hypertrophic remodeling and endothelial dysfunction-associated alterations in vascular wall elastic properties in limb muscle conduit arteries in patients with congestive HF. Poelzl et al. [14] described an intriguing relationship between phenotype changes and functional impairment in peripheral conduit arteries of HF patients. This process is very similar to what is known for ventricular remodeling in $\mathrm{HF}$.

There are several potential factors affecting aortic function in patients with HF [11]. Neurohormonal changes associated with HF include an increase in sympathetic drive and an activated renin-angiotensin system resulting in increased plasma norepinephrine levels causing vasoconstriction and sodium retention [20-23]. Angiotensin II has a hypertrophic effect on smooth muscle cells of the vascular wall [24]. Early atheromatous changes and endothelial dysfunction can also be associated with HF [25], and can affect vascular elasticity. However, there is also an interaction between aortic stiffening and HF. Aortic stiffening can increase LV load causing LV stiffening with increased wall tension, early impairment in diastolic LV relaxation, and contractility inducing LV hypertrophy and fibrosis [26, 27]. These results suggest that aortic stiffness may contribute to the progression of systolic and diastolic LV dysfunctions.

\section{Study limitations}

During this study, brachial cuff pressure measurement instead of a direct assessment of aortic pulse pressure by catheter was used. However, previous studies demonstrated an excellent correlation in aortic distensibility measured by invasive and noninvasive methods [7]. Coronary artery disease is correlated with increased aortic stiffness and was not excluded in most but not in all our patients by coronary angiography. The aortic elastic properties of control patients were somewhat larger than those described in the literature. The reason for this can be the larger body mass index $\left(29.0 \pm 5.1 \mathrm{~kg} / \mathrm{m}^{2}\right)$ in our controls. However, a relatively higher $\beta$ in the normal subjects in our study only strengthens the abnormal findings in the NCCM patients.

\section{Conclusions}

Increased aortic stiffness can be observed in patients with NCCM with moderate to severe $\mathrm{HF}$. These alterations may be due to HF-induced neurohormonal changes.

\section{References}

1. Chin TK, Perloff JK, Williams RG et al (1990) Isolated noncompaction of left ventricular myocardium: a study of eight cases. Circulation 82:507-513

2. Edwards (1995). Cardiac anatomy and examinations of cardiac specimens. In: Emmanouilides G, Allen H, Riemenschneider T, Gutgessel H (eds) Heart disease in infants, children, and adolescents. Moss and Adams, Baltimore, pp. 70-106

3. Jenni R, Oechslin E, Schneider J et al (2001) Echocardiographic and pathoanatomical characteristics of isolated left ventricular noncompaction: a step towards classification as a distinct cardiomyopathy. Heart 86:666-671

4. Oechslin EN, Attenhofer Jost CH et al (2000) Longterm follow-up of 34 adults with isolated left ventricular noncompaction: a distinct cardiomyopathy with poor prognosis. J Am Coll Cardiol 36:493-500

5. Pepine CJ, Nichols WW, Conti CR (1978) Aortic input impedance in heart failure. Circulation 58(3, pt 1):460465

6. Hundley WG, Kitzman DW, Morgan TM et al (2001) Cardiac cycle-dependent changes in aortic area and distensibility are reduced in older patients with isolated diastolic heart failure and correlate with exercise intolerance. J Am Coll Cardiol 38:796-802

7. Stefanadis C, Stratos C, Boudoulas H et al (1990) Distensibility of the ascending aorta: comparison of invasive and non-invasive techniques in healthy men and in men with coronary artery disease. Eur Heart J 11:990-996

8. Stefanadis C, Stratos C, Vlachopoulos C et al (1995) Pressure-diameter relation of the human aorta: a new method of determination by the application of a special ultrasonic dimension catheter. Circulation 92:2210-2219

9. de Laat LE, Galema TW, Krenning BJ et al (2004) Diagnosis of non-compaction cardiomyopathy with contrast echocardiography. Int J Cardiol 94:127-128 
10. Bland JM, Altman DG (1986) Statistical methods for assessing agreement between two methods of clinical measurement. Lancet 1:307-310

11. Rerkpattanapipat P, Hundley WG, Link KM et al (2002) Relation of aortic distensibility determined by magnetic resonance imaging in patients $>$ or $=60$ years of age to systolic heart failure and exercise capacity. Am J Cardiol 90:1221-1225

12. Giannattasio C, Failla M, Stella ML et al (1995) Alterations of radial artery compliance in patients with congestive heart failure. Am J Cardiol 76:381-385

13. Nakamura M, Sugawara S, Arakawa N et al (2004) Reduced vascular compliance is associated with impaired endothelium-dependent dilatation in the brachial artery of patients with congestive heart failure. J Card Fail 10:36-42

14. Poelzl G, Frick M, Huegel H et al (2005) Chronic heart failure is associated with vascular remodeling of the brachial artery. Eur J Heart Fail 7:43-48

15. Nemes A, Forster T, Csanady M et al (2004) Indices of aortic distensibility and coronary flow velocity reserve in patients with different grades of aortic atherosclerosis. Int J Cardiovasc Imaging 20:271-277

16. Nemes A, Forster T, Csanady M (2007) Impaired coronary flow velocity reserve and aortic distensibility in patients with untreated hypercholesterolemia-an echocardiographic study. Int $\mathrm{J}$ Cardiovasc Imaging 23:15-23

17. Eaton GM, Cody RJ, Binkley PF (1993) Increased aortic impedance precedes peripheral vasoconstriction at the early stage of ventricular failure in the paced canine model. Circulation 88:2714-2721

18. Khan Z, Millard RW, Gabel M et al (1999) Effect of congestive heart failure on in vivo canine aortic elastic properties. J Am Coll Cardiol 33:267-272
19. Arnold JM, Marchiori GE, Imrie JR et al (1991) Large artery function in patients with chronic heart failure: studies of brachial artery diameter and hemodynamics. Circulation 84:2418-2425

20. Zelis R, Mason DT (1970) Diminished forearm arteriolar dilator capacity produced by mineralocorticoid-induced salt retention in man: implications concerning congestive heart failure and vascular stiffness. Circulation 41:589-592

21. Zelis R, Delea CS, Coleman HN, Mason DT (1970) Arterial sodium content in experimental congestive heart failure. Circulation 41:213-216

22. Levine TB, Francis GS, Goldsmith SR et al (1982) Activity of the sympathetic nervous system and reninangiotensin system assessed by plasma hormone levels and their relation to hemodynamic abnormalities in congestive heart failure. Am J Cardiol 49:1659-1666

23. Thomas JA, Marks BH (1978) Plasma norepinephrine in congestive heart failure. Am J Cardiol 41:233-243

24. Cohn JN (1999) Pathophysiologic and prognostic implications of measuring arterial compliance in hypertensive disease. Prog Cardiovasc Dis 41:441-450

25. Kubo SH, Rector TS, Bank AJ et al (1991) Endothelium-dependent vasodilation is attenuated in patients with heart failure. Circulation 84:1589-1596

26. Kelly RP, Tunin R, Kass DA (1992) Effect of reduced aortic compliance on cardiac efficiency and contractile function of in situ canine left ventricle. Circ Res 71:490-502

27. O'Rourke MF (2001) Diastolic heart failure, diastolic left ventricular dysfunction and exercise intolerance. J Am Coll Cardiol 38:803-805 